\title{
Anomalous Thrust Production from an RF Test Device Measured on a Low-Thrust Torsion Pendulum
}

\author{
David A. Brady, Harold G. White, Paul March, James T. Lawrence, and Frank J. Davies \\ NASA Lyndon B. Johnson Space Center, Houston, Texas 77058
}

This paper describes the eight-day August 2013 test campaign designed to investigate and demonstrate viability of using classical magnetoplasmadynamics to obtain a propulsive momentum transfer via the quantum vacuum virtual plasma. This paper will not address the physics of the quantum vacuum plasma thruster, but instead will describe the test integration, test operations, and the results obtained from the test campaign.

Approximately 30-50 micro-Newtons of thrust were recorded from an electric propulsion test article consisting primarily of a radio frequency (RF) resonant cavity excited at approximately 935 megahertz. Testing was performed on a low-thrust torsion pendulum that is capable of detecting force at a single-digit micronewton level, within a stainless steel vacuum chamber with the door closed but at ambient atmospheric pressure. Several different test configurations were used, including two different test articles as well as a reversal of the test article orientation. In addition, the test article was replaced by an RF load to verify that the force was not being generated by effects not associated with the test article. The two test articles were designed by Cannae LLC of Doylestown, Pennsylvania. The torsion pendulum was designed, built, and operated by Eagleworks Laboratories at the NASA Johnson Space Center of Houston, Texas.

Approximately six days of test integration were required, followed by two days of test operations, during which, technical issues were discovered and resolved. Integration of the two test articles and their supporting equipment was performed in an iterative fashion between the test bench and the vacuum chamber. In other words, the test article was tested on the bench, then moved to the chamber, then moved back as needed to resolve issues. Manual frequency control was required throughout the test. Thrust was observed on both test articles, even though one of the test articles was designed with the expectation that it would not produce thrust. Specifically, one test article contained internal physical modifications that were designed to produce thrust, while the other did not (with the latter being referred to as the "null" test article).

Test data gathered includes torsion pendulum displacement measurements which are used to calculate generated force, still imagery in the visible spectrum to document the physical configuration, still imagery in the infrared spectrum to characterize the thermal environment, and video imagery. Post-test data includes static and animated graphics produced during RF resonant cavity characterization using the COMSOL Multiphysics ${ }^{\circledR}$ software application. Excerpts from all of the above are included and discussed in this paper.

Lessons learned from test integration and operations include identification of the need to replace manual control of the resonant cavity target frequency with an automated frequency control capability. Future test plans include the development of an automatic frequency control circuit.

Test results indicate that the RF resonant cavity thruster design, which is unique as an electric propulsion device, is producing a force that is not attributable to any classical electromagnetic phenomenon and therefore is potentially demonstrating an interaction with the quantum vacuum virtual plasma. Future test plans include independent verification and validation at other test facilities. 